\title{
Characterization of Automotive Aluminum AA356 by Photo Thermal Radiometric Spectroscopy
}

\author{
Alberto Lara Guevara ${ }^{1,2}$, Minerva Robles ${ }^{3}$, Gabriel Placencia ${ }^{3}$, Rubén Velázquez ${ }^{3}$, \\ Ignacio Rojas Rodríguez ${ }^{3 *}$ \\ ${ }^{1}$ Facultad de Informática, División de Posgrado, Universidad Autónoma de Querétaro, Querétaro, México \\ ${ }^{2}$ Facultad de Ingeniería, Postgrado en Ingeniería, Universidad Autónoma de Querétaro, Querétaro, México \\ ${ }^{3}$ Universidad Tecnológica de Querétaro, Querétaro, México \\ Email: *irojas@uteq.edu.mx
}

How to cite this paper: Guevara, A.L., Robles, M., Placencia, G., Velázquez, R. and Rodríguez, I.R. (2021) Characterization of Automotive Aluminum AA356 by Photo Thermal Radiometric Spectroscopy. Journal of Materials Science and Chemical Engineering, 9, 10-18.

https://doi.org/10.4236/msce.2021.910002

Received: September 6, 2021

Accepted: October 26, 2021

Published: October 29, 2021

Copyright ( 2021 by author(s) and Scientific Research Publishing Inc. This work is licensed under the Creative Commons Attribution International License (CC BY 4.0).

http://creativecommons.org/licenses/by/4.0/

\begin{abstract}
Photothermal radiometric spectroscopy was employed for characterizing an aluminum automotive piston with a steel insert. Amplitude and phase thermal property differences allowed the mapping of both metals. Thermal images were generated by using table stages $x-y$ movement. The thermal wave penetrated more deeply in aluminum than in steel. Results demonstrated that this relatively unknown, non-intrusively, non-contact, and non-destructive technique can be used for characterizing a wide variety of metals and other materials.
\end{abstract}

\section{Keywords}

Photothermal, Nondestructive Evaluation, Thermal Imaging, Amplitude, Phase

\section{Introduction}

Photothermal Radiometric Spectroscopy (PTR) is a powerful technique that has proven successful in characterizing the thermoelectronic properties of semiconductors and metallic materials [1] [2] [3]. Since this is a non-intrusive, non-destructive and remote-sensing methodology, it is very useful to determine effectively important properties of materials, such as thermal diffusivity, electronic carrier coefficient, minority carrier lifetime, front and back surface recombination velocities, and so on. Additionally, PTR can be utilized to assess the homogeneity and/or the presence of internal or surface defects or micro- 
cracks in metals [4]. PTR has been applied to case depth determination on heat treatment steel parts [5] and in the characterization of Steel-Cu bonded by impact [6], and in the study of thermal, structural, and microstructural properties of annealed carbon steel samples with four different carbon contents [7]. A positive correlation between Vickers microhardness and PTR amplitude images was found, in the characterization of SAE4320H Automotive Steel, indicating that the annealing process increases the PTR signal. This methodology allows the determination of the changes in the Vickers microhardness from a non-contact and remote method as in PTR [8].

PTR had been used to obtain sample thermal images of samples of Kunial brass to determine the effect of the precipitation heat treated on the metallurgical and mechanical properties [7] and were applied to, in the Thermal and Structural Characterization of Copper-Steel Bonding Interfaces Produced by Impact Welding [9], PTR had been applied in the Microstructural, Structural, and Thermal Characterization of Annealed Carbon Steels [10].

A brief review of PTR physical principles is presented in this work. Then, PTR is applied to characterize an AA356 aluminum alloy industrial piece to show the technique's versatility for analyzing and assessing the quality of a wide variety of industrial parts.

PTR is a method in which an amplitude-modulated laser beam at a certain frequency is focused onto the sample surface and scanning it. The resulting periodic heat flow in the material is a diffusion process that produces a temperature distribution called thermal wave. The black body type infrared (IR) radiation emitted by each scanned region of sample following by the periodical heating is then measured using an IR detector. In this way, PTR maps can be recorded. Physically speaking, the process for the signal generation can be described as follows: upon impinging on a sample surface, an intensity-modulated laser beam simultaneously produces direct lattice heating due to absorption, as well as a modulation in the free photo excited carrier density, in the case of free carriers (i.e., a semiconductor), provided the photon energy is greater than the band gap energy. The modulated photo-excited free carrier density depends on both the laser fluency and on the electronic properties of the material in the vicinity of the laser beam. If a wide-bandwidth IR detector, $\mathrm{HgCdTe}$, as shown in Figure 1 is focused onto a laser photo exciting spot of the sample, a superposition of IR radioactive emissions from the excited region can be then measured. This IR radiation is mainly due to the optical de-excitation of photo-excited carriers in the case of semiconductors, with the simultaneous emission of an IR photon within the blackbody (Planck) spectral range [2]. In other words, each de-exciting carrier acts as a sort of Planck radiator. In turn, these temperature variations generate "thermal waves" which can be easily detected [11]. The deep of thermal wave $(\mu)$ is determined by:

$$
\mu_{l}=\sqrt{\frac{\alpha}{\pi f}}
$$




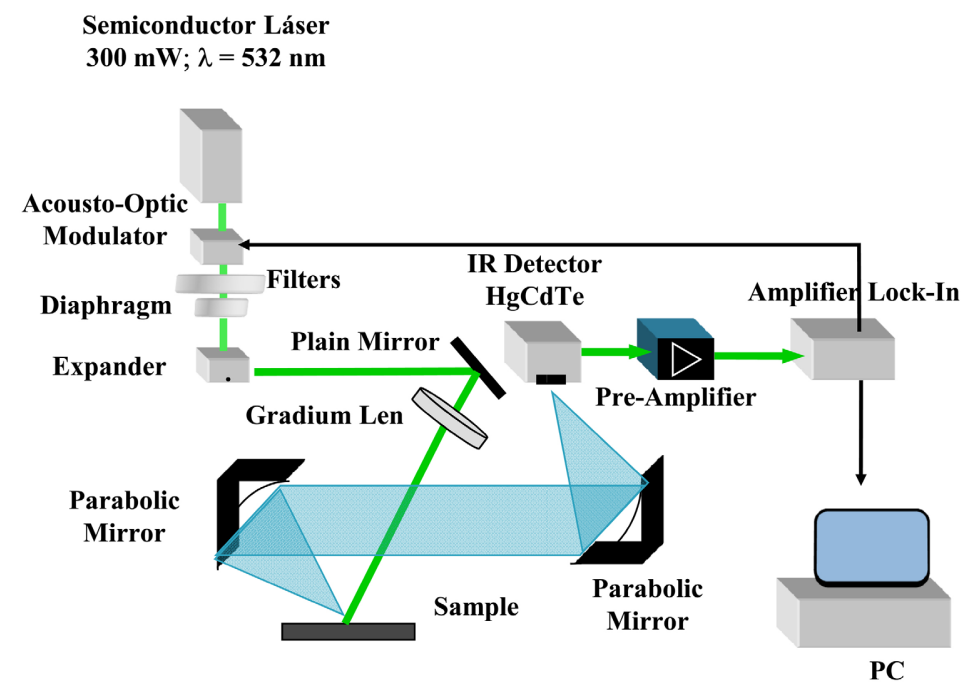

Figure 1. PTR experimental setup.

where $\alpha$ represents the thermal diffusivity and $f$ the frequency modulation.

If the sample is structurally uniform, thermal diffusion length must be the same at any point located on the surface. PTR amplitude and phase signals of all points under the same conditions have to be detected in the same way by the IR detector. It is interesting to notice that, at high frequencies, the thermal wave is located very close to the surface, so it is possible to detect variations in the surface recombination rate. According to Shockley and Read, the lifetime is an effective parameter that depends on both the surface and bulk phenomena [12]. Therefore, in agreement with the radiometric theory, intermediate range frequencies are required to observe changes in this parameter [1] [2]. On the other hand, thermal diffusivity is a bulk property, so the corresponding variations must be observed at relatively low frequencies. Thus, the PTR signal can be written as:

$$
S_{R}=C_{T} S_{T}+C_{p} S_{p}
$$

where $S_{R}$ is the PTR signal, $S_{T}$ is the thermal component and, $S_{p}$ represents the plasma contribution, $C_{T}$ y $C_{p}$ are the weight parameters for both contributions.

In the case of metals with the contrast between different regions, differences could be attributed to thermal and optical properties of the involved metals. PTR images reveal the morphological aspects of the interfaces. It has been demonstrated for the first time that PTR is a useful technique to characterize nonmetallic and metallic inclusions considered impurities in most metals. The analyzed AA356 aluminum alloy is widely used in the automotive industry, which properties and microstructural characteristics are significantly different from steel, as well as thermal properties.

\section{Material and Methods}

\subsection{Sample Preparation}

An AA356 aluminum alloy piston produced by the permanent mold process was 
analyzed as shown in Figure 2. The piston had a carbon steel insert which was placed in the mold before the cast process. The insert was used as a cooler during the cast and as mechanical reinforce. The typical chemical composition of AA356 aluminum alloy is: $90.2 \%-93.3 \% \mathrm{Al}, 0.25 \%-0.45 \% \mathrm{Mg}$ and $6.5 \%-7.5 \%$ Si. The aluminum piston sample was metallographically prepared.

\subsection{Experimental Setup}

The experimental setup for PTR imaging is shown in Figure 1. This configuration has already been described in detail [1] [2] [3]. The setup consisted of a 300 $\mathrm{mW}$ semiconductor laser currently-modulated. The beam was expanded, collimated and then focused onto the surface of the sample. The harmonically modulated IR Planck radiation from the optically excited sample surface was collected by two off-axis parabolic mirrors. The spot-size of the focused gaussian laser beam was approximately $40 \mu \mathrm{m}$ in diameter, which was attained by using a gradium glass lens with $12.4 \mathrm{~cm}$ focal distance. The IR emission was collected by the off-axis parabolic mirrors and focused onto a CdTeHg IR detector, liquidnitrogen-cooled with spectral response from 2 to $12 \mu \mathrm{m}$. The output signal was pre-amplified and fed to a lock-in amplifier and the data was processed in a personal computer. The excitation beam was modulated from $10 \mathrm{~Hz}$ to $100 \mathrm{kHz}$ via an acousto-optic modulator (AOM).

\subsection{PTR}

In this job, PTR was used to characterize the automotive piston sample. Three points of the aluminum area and 3 points of the steel insert area were tested at $10 \mathrm{~Hz}$ to $100 \mathrm{kHz}$ modulation frequency as shown in Figure 3. Major amplitude and phase differences between aluminum and steel were observed at $10 \mathrm{kHz}$ frequency. Thus, $10 \mathrm{kHz}$ frequency was used to obtain thermal images over a $2000 \mu \mathrm{m} \times 6000 \mu \mathrm{m}$ area, each $60 \mu \mathrm{m}$. Amplitude and phase thermal property differences allowed the mapping of both metals. PTR amplitude and phase thermal images were generated by using table stages $\mathrm{x}$-y movement.

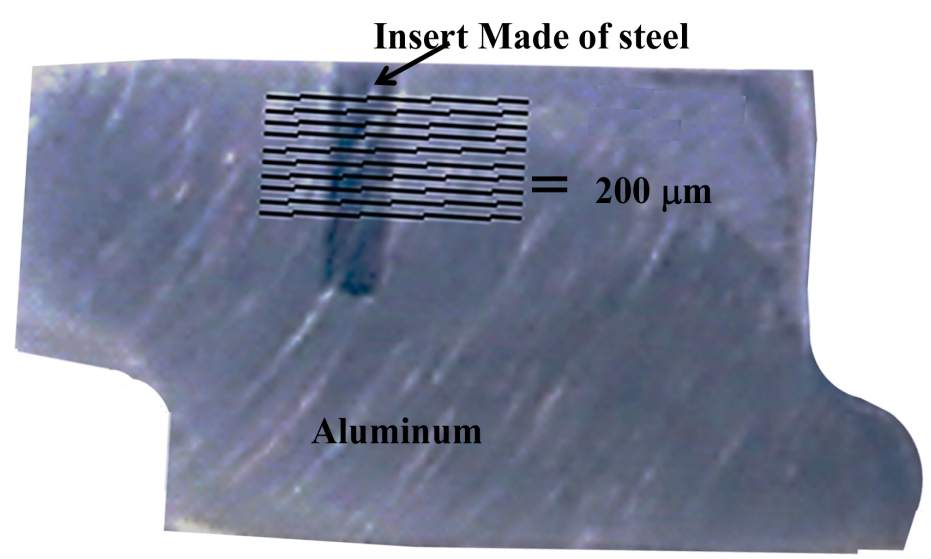

Figure 2. AA356 aluminum alloy automotive piston sample with a steel insert at the characterized section. 


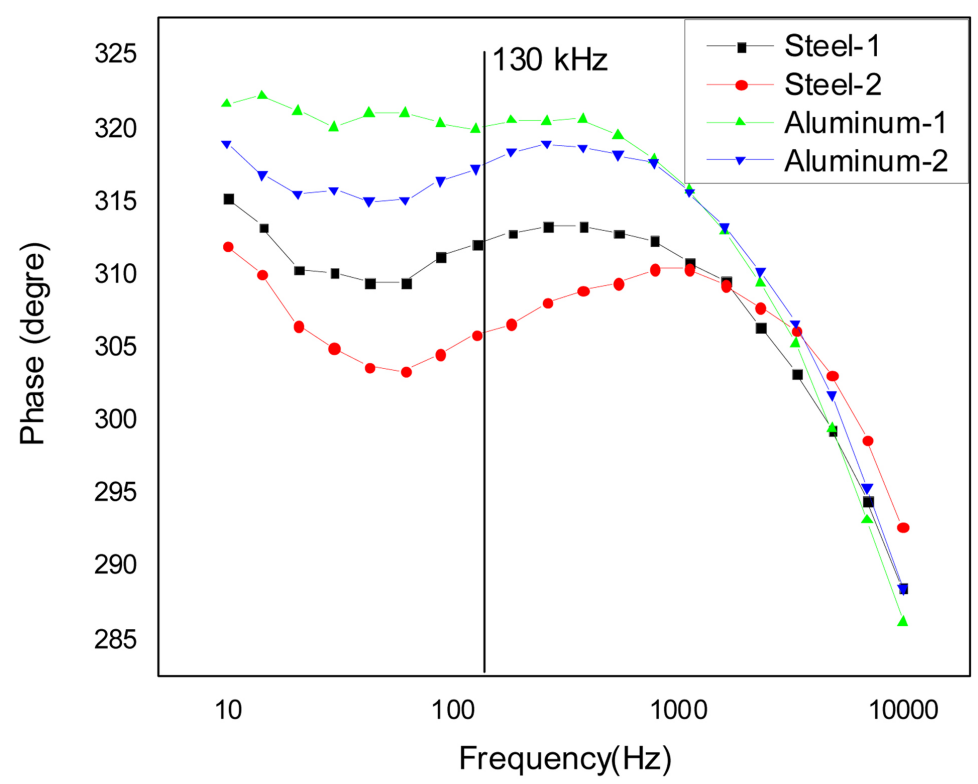

(a)

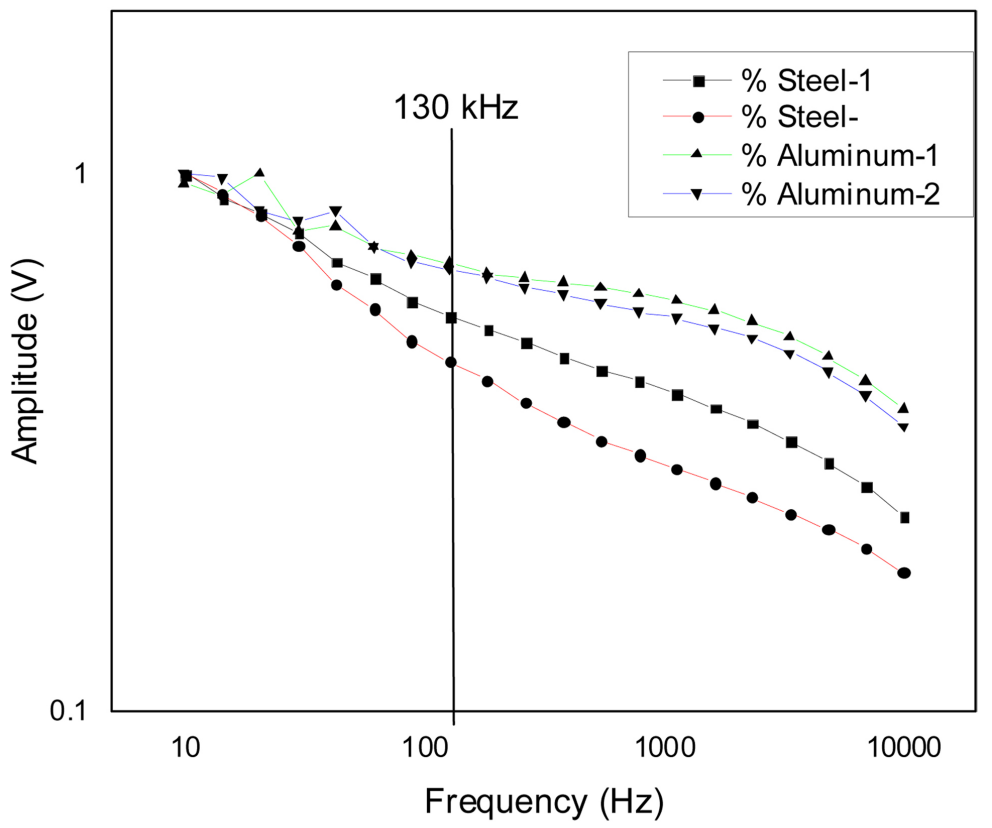

(b)

Figure 3. PTR amplitude (a) and phase (b) spectra to determine the frequency to generate thermal images.

\section{Results and Discussion}

Figure 4(a) shows the PTR amplitude thermal image of a steel insert as a heat dissipation part of an aluminum automotive piston. It is known that steel thermal diffusivity is much smaller than aluminum thermal diffusivity $\left(0.227 \mathrm{~cm}^{2} / \mathrm{s}\right.$ and $0.968 \mathrm{~cm}^{2} / \mathrm{s}$, respectively) [13]. It is well known that the radiometric signal is proportional to the surface emission, and also, that it is proportional to the thermal diffusivity. Thus, the highest values corresponded to aluminum. Figure 4(a) shows that the amplitude signal depended on aluminum and steel thermal 


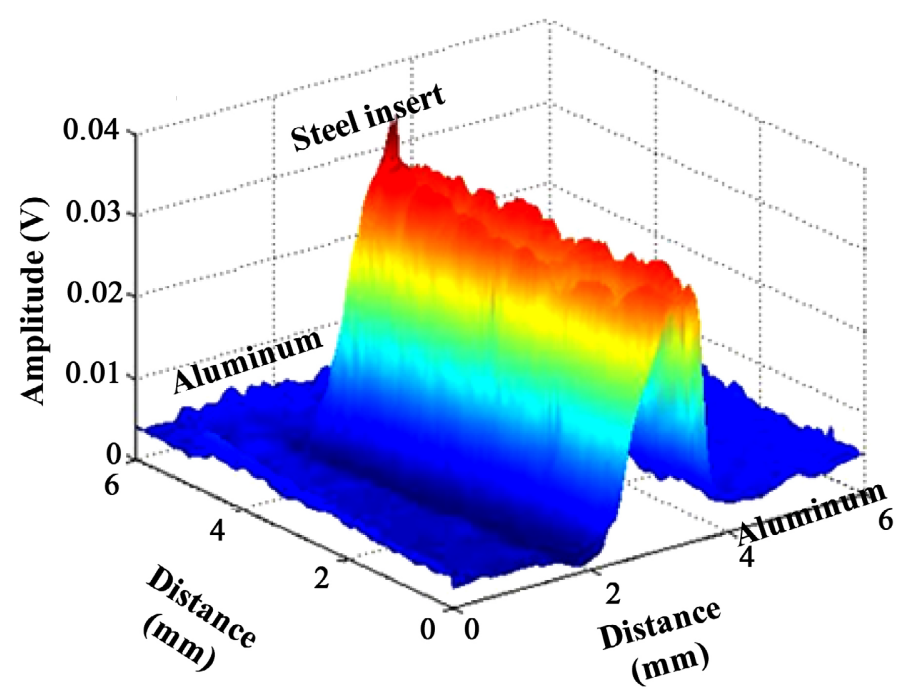

(a)

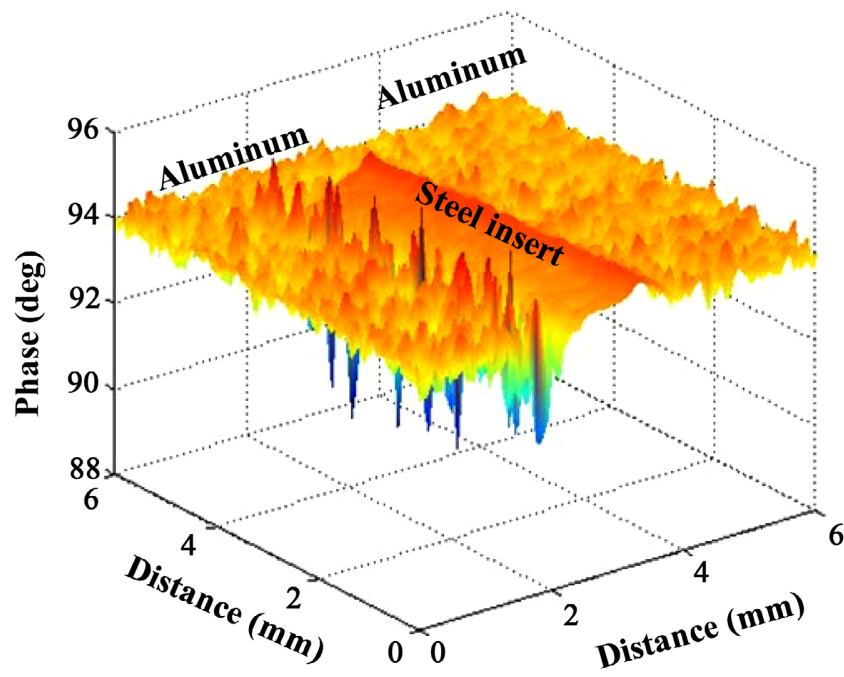

(b)

Figure 4. Thermal images of piston sample. (a) Amplitude; (b) Phase.

properties, since thermal conductivity is exclusive of lattice processes [14]. Also, Figure 4(a) shows that amplitude corresponded to a low thermal diffusivity in the steel insert region. In the case of phase thermal images illustrated in Figure 4 (b) exists a gradient of at least 25 degrees. This gradient shows a very good PTR sensitivity to determine inclusions in metals, taking advantage of the thermophysical property differences (thermal diffusivity and conductivity). Thermal properties lead to discrepancies in amplitude and phase measurements, allowing to differentiate materials. According to [11], the amplitude thermal wave that is generated by aluminum and steel due to the absorption of modulate laser radiation is described by:

$$
T(x, t)=\frac{I_{0}}{2 \varepsilon \sqrt{\omega}} \exp \left(-\frac{x}{\mu}\right) \cos \left(\omega t-\frac{x}{\mu}+\frac{\pi}{4}\right)
$$

The first term of Equation (3) is a constant parameter depending on the fre- 
quency, $\omega=2 \pi f$ and $f$ is the modulation frequency, $I_{0}$ is the laser intensity, $x$ is the sample thickness, and $\varepsilon$ is the thermal effusivity. These parameters determined the temperature variation amplitude at the sample surface. The reflection coefficient (absorption) for wavelength $532 \mathrm{~nm}$ was different between aluminium and steel. In Figure 4(a) the heat conversion radiation was lower in steel than in aluminum, and thus, the PTR amplitude signal was lower in steel than in aluminum.

Equation (1) determines the damping of the thermal wave as well as the phase lag between the excitation and the thermal response (thermal diffusion length and thermal diffusivity). As the modulation was a fixed frequency, the measured PTR amplitude signal was proportional to the reciprocal of the thermal effusivity; while the PTR phase was proportional to the $x / \mu$ term, Equation (4) and Equation (5). Thermal effusivity was higher in aluminum than in steel. Thermal wave amplitude was higher at the aluminum region than in the steel part, as is shown in Figure 4(a).

In the case of the phase signal illustrated in Figure 4(b), the highest PTR signal belonged to aluminum, while the lowest one corresponded to steel. These results reflected that the thermal diffusivity was higher in aluminum than in steel. The PTR phase can be described as follows:

$$
\begin{gathered}
\phi=\frac{x}{\mu}+\phi_{o} \\
\phi=x \sqrt{\frac{\pi f}{\alpha}}+\phi
\end{gathered}
$$

Here $\alpha$ is the sample thermal diffusivity. According to Equation (4) and Equation (5), if the highest thermal diffusivity coefficient corresponds to aluminum, this means that the aluminum PTR signal is lowest. Then, the thermal wave penetrated more deeply in aluminum than in steel.

It is important to know that the radiometric signal strongly depends on the surface finished [11], so it can be utilized to detect surface damage, assess the state of a protecting coating or measure degradation of materials.

\section{Conclusions}

PTR results have shown that this technique has a good potential to characterize different metals.

Homogeneity analysis of metallic materials can be effectively obtained by PTR.

The steel insert in an aluminum piece of this work clearly revealed that changes in amplitude PRT signal corresponded to regions with different dendritic structures, caused by local variations of solidification rates.

This technique can also be applied to other metals in order to determine the content of an alloy without affecting its other properties, to determine thin layers, and in the characterization of metals parts heat treated. 
Finally, the possibilities of this technique for quality assessment of a variety of materials, including organic ones, are an open field to research.

\section{Conflicts of Interest}

The authors declare no conflicts of interest regarding the publication of this paper.

\section{References}

[1] Rodríguez, M.E., Mandelis, A., Nicolaides, L., Pan, G., García, J. and Riopel, Y. (2000) Computational Aspects of Laser Radiometric Multiparametric Fit for Carrier Transport Property Measurements in Si Wafers. Journal of Electrochemical Society, 147, 687-698. https://doi.org/10.1149/1.1393254

[2] Mandelis, A. (1998) Laser Infrared Photothermal Radiometry of Semiconductors: Principles and Applications to Solid-State Electronics. Solid-State Electronics, 42, 1-15. https://doi.org/10.1016/S0038-1101(97)00238-4

[3] Ikari, T., Salnick, A. and Mandelis, A. (1999) Theoretical and Experimental Aspects of Three Dimensional Infrared Photothermal Radiometry of Semiconductors. Journal of Applied Physics, 85, 7392-7397. https://doi.org/10.1063/1.369368

[4] Kaufman, I., Chang, P.T., Hsu, H.S., Huang, W.Y. and Shyong, D.Y. (1987) Photothermal Radiometric Detection and Imaging of Surface Cracks. Journal of Nondestructive Evaluation, 6, 87-100. https://doi.org/10.1007/BF00568887

[5] Wanga, C. and Mandelis, A. (2007) Case Depth Determination in Heat-Treated Industrial Steel Products Using Photothermal Radiometric Interferometric Phase Minima. NDT \& E International, 40, 158-167.

https://doi.org/10.1016/j.ndteint.2006.09.005

[6] Rojas-Rodriguez, I., Jaramillo-Vigueras, D., Velázquez-Hernández, R., Del Real, A., Serroukh, I., Baños, L., García, J. and Rodríguez-García, M.E. (2008) Thermal and Structural Characterization of Copper-Steel Bonding Interfaces Produced by Impact Welding. Journal of Materials and Manufacturing Processes, 23, 823-828. https://doi.org/10.1080/10426910802384672

[7] Rojas-Rodríguez, I., Lara-Guevara, A., Salazar-Sicacha, M., Mosquera-Mosquera, J.C., Robles-Agudo, M., Ramirez-Gutierrez, C. and Rodríguez-García, M. (2018, April) The Influence of the Precipitation Heat Treatment Temperature on the Metallurgical, Microstructure, Thermal Properties, and Microhardness of an Alpha Brass. Materials Sciences and Applications, 9, 440-454. https://doi.org/10.4236/msa.2018.94030

[8] Lara-Guevara, A., Rojas-Rodríguez, I., Velazquez-Hernandez, R., et al. (2017) Photothermal, Structural, and Microstructural Characterization of SAE4320H Automotive Steel. Journal of Materials Engineering and Performance, 26, 2040-2046. https://doi.org/10.1007/s11665-017-2633-7

[9] Lara-Guevara, A., Rojas-Rodríguez, I., Ortiz-Echeverri, C.J., Robles-Agudo, M. and Rodríguez-García, M.E. (2017) Thermal, Structural, and Microstructural Characterization of Eutectoid Steel at Different Heat Treatments. Journal of Materials Research, 32, 2202-2209. https://doi.org/10.1557/jmr.2017.47

[10] Lara-Guevara, A., Ortiz-Echeverri, C.J., Rojas-Rodriguez, I., et al. (2016) Microstructural, Structural, and Thermal Characterization of Annealed Carbon Steels. International Journal of Thermophysics, 37, Article No. 99.

https://doi.org/10.1007/s10765-016-2105-6 
[11] Mandelis, A. (2000) Thermal Waves and its Applications. Physics Today, 29.

[12] Shockley, W. and Read, W.T. (1952) Statistics of the Recombination of Holes and Electrons. Physical Review, 87, 835-842. https://doi.org/10.1103/PhysRev.87.835

[13] Tolukian, Y.S., Powell, R.W., Ho, C.Y. and Nicolaou, M.C. (1973) Thermophysical Properties of Matter. The TPRC Data Series, 10, $51 \mathrm{p}$.

[14] Bhandari, C.M. and Rowe, D.M. (1998) Thermal Conduction in Semiconductor. John Wiley \& Sons, New York, 47-49. 